\title{
Does One Bicycle Facility Type Fit All? Evaluating the Stated Usage of Different Types of Bicycle Facilities among Cyclists in Quebec City, Canada
}

Transportation Research Record 2019, Vol. 2673(6) 650-663

(C) National Academy of Sciences: Transportation Research Board 2019 Article reuse guidelines: sagepub.com/journals-permissions DOI: $10.1177 / 036|1981| 984474$ | journals.sagepub.com/home/trr (SSAGE

\author{
Marie-Pier Veillette', Emily Grisé', and Ahmed El-Geneidy'
}

\begin{abstract}
For cities wishing to foster a strong culture of cycling, developing a network of safe and efficient bicycle infrastructure is paramount, yet not a straightforward task. Once transport professionals have selected the optimal location for a new bicycle facility, determining the optimal facility type is imperative to ensure that the new infrastructure encourages cycling trips and increases the safety of cyclists. The present study presents a nuanced approach to evaluating cyclists' usage of various types of bicycle facilities. To achieve this goal, we employed survey data of cyclists in Quebec City, Canada, to study how many cyclists reported using a particular bicycle facility in the survey against their reasonable access to those reported facilities. To account for different preferences, behavior, and motivations among cyclists, we segmented our study sample into six types of cyclist. Finally, regression modeling was employed to predict the stated usage of three facility types present in the study area (recreational path, bi-directional protected lane, and painted lane), while controlling for access to this path, cyclist type, and personal and neighborhood characteristics. Results indicate that if a cyclist has access to each facility type on their commute, they are most likely to use a recreational path on their commute, followed by a painted bicycle lane. Respondents with access to a bi-directional lane are no more likely to report using this facility than respondents without access. Overall, this study is intended to encourage a dialog between cyclists and transport practitioners to uncover the factors contributing to effective bicycle infrastructure.
\end{abstract}

Should I cycle to work today or not? Although one can answer this question by simply saying yes or no, the reasons for mobility behavior are far more convoluted. Recent studies have identified a bundle of factors explaining why an individual is willing to cycle, including the presence of bicycle parking at a destination, access to bicycle facilities, travel distance, perceived and real safety, physical and social environment, weather conditions, and attitudes (1-7).

Bicycle facilities with greater separation from motorized traffic are recognized as preferable by many cyclists $(4,8,9)$. As the construction of physically separated bicycle lanes generally requires higher capital investments than other facility types, it is essential to ensure that the best design is implemented to deliver a return on investment, in relation to encouraging cycling trips and increasing the safety of cyclists. Several municipalities measure the performance of a new facility by comparing the number of cyclists using a street before and after the construction of a facility or with estimated usage levels. However, these measures do not consider the behavior of cyclists, and choices they make regarding the use of a particular bicycle facility. Evaluating the choice of cyclists to use various bicycle facilities would provide planners and policymakers with a nuanced perspective of how cyclists perceive different facility types. Accordingly, this study presents a new method of evaluating bicycle facility usage in Quebec City, Canada. Using a survey conducted by the Transportation Research at McGill group in 2015, we evaluate what facilities cyclists have reasonable access to during their commute to work or school and compare this with which facility types each cyclist reported using, and thus measure which facility types are not well used by cyclists. Furthermore, we take this method one step further by segmenting cyclists according to their behavior, attitudes, and preferences to better understand

\footnotetext{
'School of Urban Planning, McGill University, Montréal, QC, Canada
}

Corresponding Author:

Address correspondence to Ahmed El-Geneidy:

ahmed.elgeneidy@mcgill.ca 
whether certain groups of cyclists with similar characteristics are more or less willing to cycle on different paths.

This paper is divided in four sections. We will first explore the existing literature on cyclists' segmentation techniques and the preferences of cyclists toward different bicycle facilities. This will be followed by an analysis section, where we begin by describing the data employed in this paper, which is followed by a detailed overview of the methods used in our analysis, and we then present the findings of our study. Finally, we provide recommendations and highlight the implications of our results.

\section{Literature Review}

\section{Segmentation Approaches}

Cyclists are not a homogenous group of individuals (1013). Studies have revealed that among cyclists, unique groups are distinguishable according to their cycling facility preferences, motivations, experience, habits, and so forth $(10,14)$. Segmentation techniques are commonly employed to acknowledge that policies will not affect all groups of users (for example public transit users) or individuals (in this case cyclists) in the same way. Performing a cyclist segmentation can help practitioners uncover groups of cyclists with similar behavioral and attitudinal patterns. After identifying distinct groups of cyclists in a region, practitioners can design strategies that suit the motivations, needs, and desires of these different groups, thereby increasing their probability of success and ensuring that limited resources are targeted effectively (15).

There are two main segmentation approaches that are commonly applied by both cycling researchers and practitioners. The first approach is to determine a priori the number and types of cyclists before analyzing a dataset, to thereafter make cyclists fit into these pre-defined categories. Geller's well-known and widely used typology was created in this fashion (13). Geller's four types of cyclists are: no way no how; interested but concerned; enthused and confident; and strong and fearless. This segmentation approach includes both non-cyclists and cyclists, which can be somewhat confusing. Dill and McNeil $(11,12)$ examined the suitability of Geller's segmentation, a first time at the Portland regional scale, and a second time at nationwide levels in US urban areas. The authors' conclusions tend to support the idea that one unique cyclist typology can fit all urban contexts, despite acknowledging differences in cycling facility supplies and in the modal split between the areas studied. Generalizing one approach to all urban settings is likely not a guarantee of success and, in fact, there may be other distinct groups of cyclists present in a region, as observed by Damant-Sirois, Grimsrud, and ElGeneidy (10).
The second segmentation approach commonly employed by researchers utilizes empirical techniques such as factor analysis followed by $K$-means clustering. Factor analysis is commonly used to derive factors of related data or questions and is particularly important when working with a large set of correlated questions. $K$ means cluster analysis is then employed to uncover how these factors relate to identify distinct groups of individuals. These techniques are commonly utilized when working with large survey datasets $(16,17)$. Studies specific to cyclists that employed this technique include Gatersleben and Haddad (18) who found four types of cyclist in England: responsible cyclists; lifestyle cyclists; commuter cyclists; and hippie-go-lucky cyclists. Eriksson, Friman and Gärling (14) used the 2009 City of Nanjing, China's Household survey to create six distinct types of commuter cyclist according to factors including their willingness to cycle, need for fixed schedule, desire for comfort, and environmental awareness. Recommendations drawn from this study included cycling facility improvements tailored to each type of cyclist, such as improving network connectivity, increasing the cycling network density, and better integrating cycling facilities with land use. Finally, Damant-Sirois, Grimsrud, and El-Geneidy (10) identified the following four groups of cyclists living in Montreal, Canada: dedicated cyclists; path-using cyclists; fairweather utilitarians; and leisure cyclists. These cyclists were segmented according to factors such as stated cycling facility preference, motivation, and social encouragement.

\section{Usage and Preferences for Bicycle Facilities}

There are different types of bicycle facilities as thoroughly defined in $(1,4)$. As it appears widely accepted that cycling facilities are a key component to encouraging cycling, many researchers have sought to uncover key knowledge regarding which type of cycling facility can best help cities achieve this goal. For example, many studies have evaluated the routes cyclists take to shed light on the proportion of cyclists who divert to use a bicycle facility (19-23).

In Vancouver, Canada, Winters et al. (23) observed the ratio of the distance of stated cycling routes to the shortest route distance. The authors observed modest detour levels $(360 \mathrm{~m})$, and according to cyclists these detours were for reasons such as to avoid arterial roads and use local roads instead, off-street paths, and routes with bicycle facilities. Using a large-scale survey of cyclists in Montreal, Larsen and El-Geneidy (21) used distance decay functions to understand how far cyclists will travel to use different bicycle facilities, where it was evident that all cyclists will travel longer distances to use off-street bicycle facilities compared with all other facility 
types, however this preference was more pronounced among infrequent cyclists. However, after considering which factors are most influential for a cyclist's route choice, they observed that the diversion distance to a nearby facility is best explained by the supply of nearby facilities and the distance traveled on a given facility. This suggests that widespread implementation of longer, continuous cycling facilities may ultimately attract more users. In Portland, Oregon, USA, Broach, Dill and Gliebe (8) used GPS units to observe the travel behavior of frequent cyclists, finding that the following factors are important in the route choice of cyclists: distance, turn frequency, slope, traffic signals, and traffic volume. The authors constructed a route choice model to compare the selected route of each cyclist to many route alternatives, and the findings suggest that individuals cycling regularly preferred off-street separated bicycle paths and bicycle boulevards over stripped lanes and arterial bike lanes, as using these two facilities diminishes traffic exposure. These findings differ from several stated preference surveys that found experienced cyclists preferred cycling without designated facilities $(10,24,25)$. The authors also recognize the importance of the local context. The network of separate paths present in Portland is potentially more extensive and/or located to facilitate utilitarian trips compared with other contexts that may not link utility trip origins and destinations, therefore presenting the importance of practicing caution when comparing results of studies of this nature. More recently, Broach and Dill (26) found that the presence of off-street bicycle paths, bike boulevards or routes enabling cyclists to avoid motorized traffic influenced cyclists to use a particular route where these facilities were available. The authors accordingly recommend investments in cycling infrastructure with traffic separation or in low-traffic streets.

Preferences for cycling facilities also vary among cyclists based on gender and age. Aldred et al. (27) observed a greater gender balance on protected bicycle lanes in London, UK, and suggests that protected cycle infrastructure may help to 'normalize' the image of cycling, such as the case of bicycle sharing systems in London (28). Similarly, in a systematic review of stated preference studies that evaluated infrastructure preferences by age and gender, Aldred et al. (27) found that women had stronger preferences for bicycle infrastructure with greater separation compared with men. As per age, more than half of the studies examined did not observe a preference among older adults toward separation from motor vehicles. No groups studied however stated a preference for cycling in motorized traffic. Conversely, revealed preference studies did not find significant differences in facility usage by gender $(8,21)$. However, revealed preference studies (for example observing route choice and facility usage counts) do not necessarily reflect the active choice or preference of that facility type over another, but may rather reflect their best option to reach their destination (27). In reality, physical characteristics, design, location, and the condition of bicycle facilities may be different according to specific urban settings (4) which can partly explain these contrasting findings.

It would appear from this brief overview of the literature on cyclist typologies and preferences for bicycle facilities that carefully considering local context is imperative for effective bicycle planning. For this reason, designing the best facility is more complicated than selecting the desired level of separation from vehicle traffic. Rather, factors such as the length of the facility, proximity to destinations, changes in elevation, traffic signals, and vehicle traffic all play a role in affecting how cyclists will perceive and utilize a bicycle facility. In this study, we present a method to examine what types of facilities bicyclists reported using, and how this compares with the approximated route from their home to their work location. In other words, we want to focus on which facilities are nearby a cyclist's daily commuting route and to determine the odds of their using this facility. Furthermore, we evaluate how different groups of cyclists react to the presence of different facility types near their routes. The grouping of cyclists will be derived from a cycling survey conducted in Quebec City in 2015, to identify types of cyclists that have similar attitudinal and behavioral patterns.

\section{Data}

This study employs data collected in the 2015 Quebec City Bicycling Travel Survey conducted by the Transportation Research at McGill group in collaboration with the City of Quebec. The data were collected through an online survey between August and October 2015. Invitations to participate were sent by email to Quebec City employees for testing the survey. Furthermore, the survey was advertised in local newspapers, and the following groups were invited to share the online survey: university student associations; the public transit agency serving Quebec City; local associations; post-secondary institutions; cyclist advocacy groups; and school boards. In addition, an article in the local newspaper was written by a local journalist encouraging participation in the survey. Also, various social media groups announced the survey online on Facebook and Twitter.

A total of 1,823 full responses were collected, with the majority of respondents being utilitarian cyclists. For the purpose of this study, we excluded respondents who did not provide a home, workplace, or school geographic 
Table I. Bicycle Facility Characteristics per Borough

\begin{tabular}{|c|c|c|c|}
\hline Bicycle facilities characteristics & $\begin{array}{l}\text { Recreational } \\
\text { path }\end{array}$ & $\begin{array}{l}\text { Bi-directional path } \\
\text { with median }\end{array}$ & $\begin{array}{l}\text { Painted lane } \\
\text { (one way) }\end{array}$ \\
\hline Total length $(\mathrm{km})$ & 76.39 & 65.37 & I I 2.84 \\
\hline \multicolumn{4}{|c|}{ Percentage of facilities adjacent/or located on streets with the following speed limit (\%) } \\
\hline $50 \mathrm{~km} / \mathrm{h}$ & - & 45.8 & 48.74 \\
\hline $60 \mathrm{~km} / \mathrm{h}$ & - & 51.9 & 43.24 \\
\hline $80 \mathrm{~km} / \mathrm{h}$ & - & 2.3 & 7.97 \\
\hline $\begin{array}{l}\text { Number of intersections divided } \\
\text { by cycling facility length }(\mathrm{km})\end{array}$ & 1.80 & 2.5 & 4.46 \\
\hline $\begin{array}{l}\text { Retail, commercial, and institutional } \\
\text { activities density within a } 500 \mathrm{~m} \\
\text { buffer around each facility types }\end{array}$ & 139 per km² & 158 per km² & 84 per km² \\
\hline \multicolumn{4}{|l|}{ Total length per borough $(\mathbf{k m})^{\mathrm{a}}$} \\
\hline Beauport & 18.75 & 5.62 & 6.16 \\
\hline Charlesbourg & 5.68 & 5.59 & 11.98 \\
\hline La Cité-Limoilou & 15.90 & 4.38 & 4.40 \\
\hline La Haute-Saint-Charles & 13.25 & 2.88 & 28.11 \\
\hline Les Rivières & 10.32 & 16.59 & 15.40 \\
\hline Sainte-Foy-Sillery-Cap-Rouge & 12.15 & 29.67 & 33.63 \\
\hline Outside City limits & 0.34 & 0.64 & 13.15 \\
\hline
\end{tabular}

${ }^{\mathrm{a}}$ The location of each borough can be found in Figure I.

location (e.g., postal codes or a nearby intersection that was provided on a map). Additionally, we omitted respondents who cycled for recreation and grocery shopping because we did not have access to the location of their destinations. Moreover, we did not consider respondents who cycled less than $1 \mathrm{~km}$ to reach their workplace or school. As a result, our sample consists of 877 home and work/school cycling trips.

Survey questions were designed to identify the needs, motivations and deterrents of cyclists and non-cyclists residing in Quebec City. The main survey questions of interest to this study were the importance of various factors in cyclists' decisions to cycle and whether or not each respondent reported using each facility (recreational path, bi-directional path, and painted lane). Each bicycle facility type included in the survey was accompanied by a picture to ensure that respondents associated the right facility type to each question. The main distinguishing differences in these facilities reside in their level of separation from traffic, where recreational paths are off-street trails found mainly in parks, bi-directional paths are physically separated from traffic by a median, and painted lanes are recognizable by on-street painted road markings. The distribution of each of these facilities is presented in Figure 1, and the characteristics of the bicycle facilities is presented in Table 1. Our study area has approximately $76 \mathrm{~km}$ of recreational paths, $65 \mathrm{~km}$ of bidirectional paths separated by a median, and $112 \mathrm{~km}$ of painted lanes. Note that other types of cycling facilities are present but are not being considered in the present research.
Interestingly, slightly more than half of bi-directional paths are adjacent to arterial roads with a speed limit of $60 \mathrm{~km} / \mathrm{h}$. In a similar vein, $8 \%$ of painted lanes are located on major roads with a maximum speed limit of $80 \mathrm{~km} / \mathrm{h}$, whereas only $2.3 \%$ of bi-directional paths are located on these high-speed arterials. In relation to connectivity, painted lanes intersect with the street grid almost 2.5 times more often than recreational paths and 1.7 times more than bi-directional paths. Furthermore, the density of retail, commercial, and institutional uses, $500 \mathrm{~m}$ on each side of bi-directional paths, is almost twice as high compared with painted lanes. Finally, unlike the two other types of facilities, bi-directional paths are mostly concentrated in two boroughs: Les Rivières and Sainte-Foy-Sillery-Cap-Rouge.

\section{Analysis}

Our analysis consists of a three-step procedure (Figure 2). Each step of our analysis is conducted using the aforementioned sample of cyclists. First, we performed spatial analysis using ArcGIS to determine what types of bicycle facilities each respondent has reasonable access to when commuting to work or school. Second, we carried out a factor analysis followed by a $K$-means cluster analysis to segment cyclists into distinct groups according to their motivations, childhood characteristics and cycling habits. Finally, three logistic regression models were constructed to predict the usage each the three types of bicycle facilities (recreational paths, bi- 


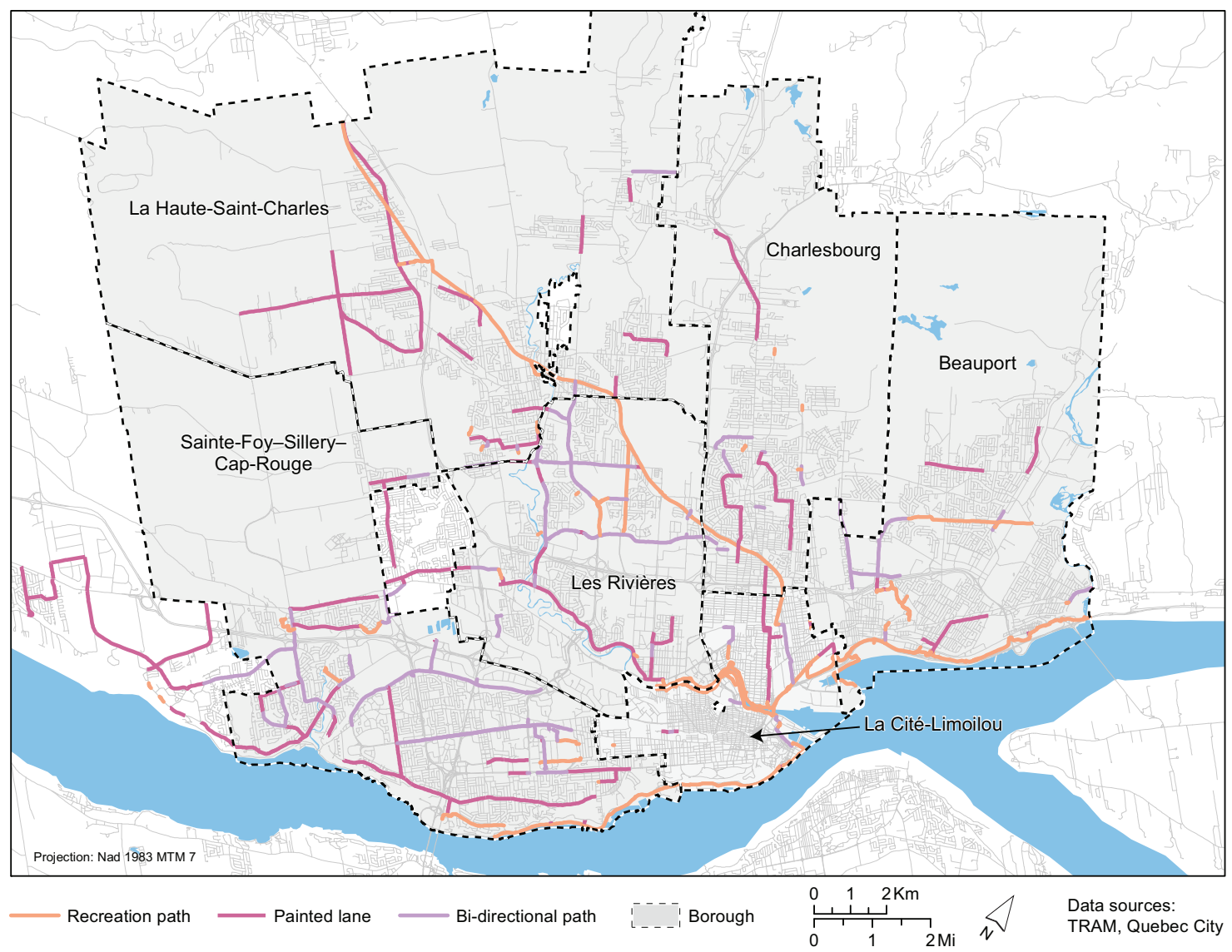

Figure I. Location of recreational paths, bi-directional paths, and painted lanes in Quebec City, Canada.

directional paths, and painted lanes), while having reasonable access to these facility types.

\section{Spatial Analysis}

The first step of our spatial analysis involved routing each cyclist's trip. Each respondent provided their home postal code and the coordinates of their work or school location. Note that in Canada a postal code is a geographic area smaller than a census tract, which is comparable to the size of half a city block, thus enabling a finer scale analysis. Using this information, we modeled the shortest route from home (origin) to work or school (destination) using Network Analyst 'Closest Facility' Tool and a street network containing all segments potentially usable by cyclists. Only highways were thereby removed from the street network.

Next, we generated a network buffer around each route, to determine which types of bicycle facilities each respondent had access to, with the assumption that cyclists are willing to divert from their shortest path to use a preferred facility. Consistently the literature demonstrates that cyclists choose to deviate from the shortest path to use bicycle facilities $(20,29-31)$, however diversion distances have been found to be best explained by facility length and supply of nearby facilities (21) as well as by type of cyclist (10). In one study, a detour was calculated to measure how far a cyclist will travel from their shortest route to reach a bicycle facility and found that, of cyclists who use a bicycle facility, on average they will detour $12 \%$ or $695 \mathrm{~m}$ (32). In our study we applied a conservative $10 \%$ diversion rate, assuming that cyclists are willing to detour a distance up to $10 \%$ of their route to use a preferred facility.

To generate the network buffer, we converted each respondent's route into several points equally distanced. In ArcGIS Network Analyst's Service Area, we computed a buffer following the street grid around all points, where sizes were set according to the route's diversion rate previously calculated. Finally, buffers around points forming one route were merged together to form a single buffer for each route. An example network buffer is 


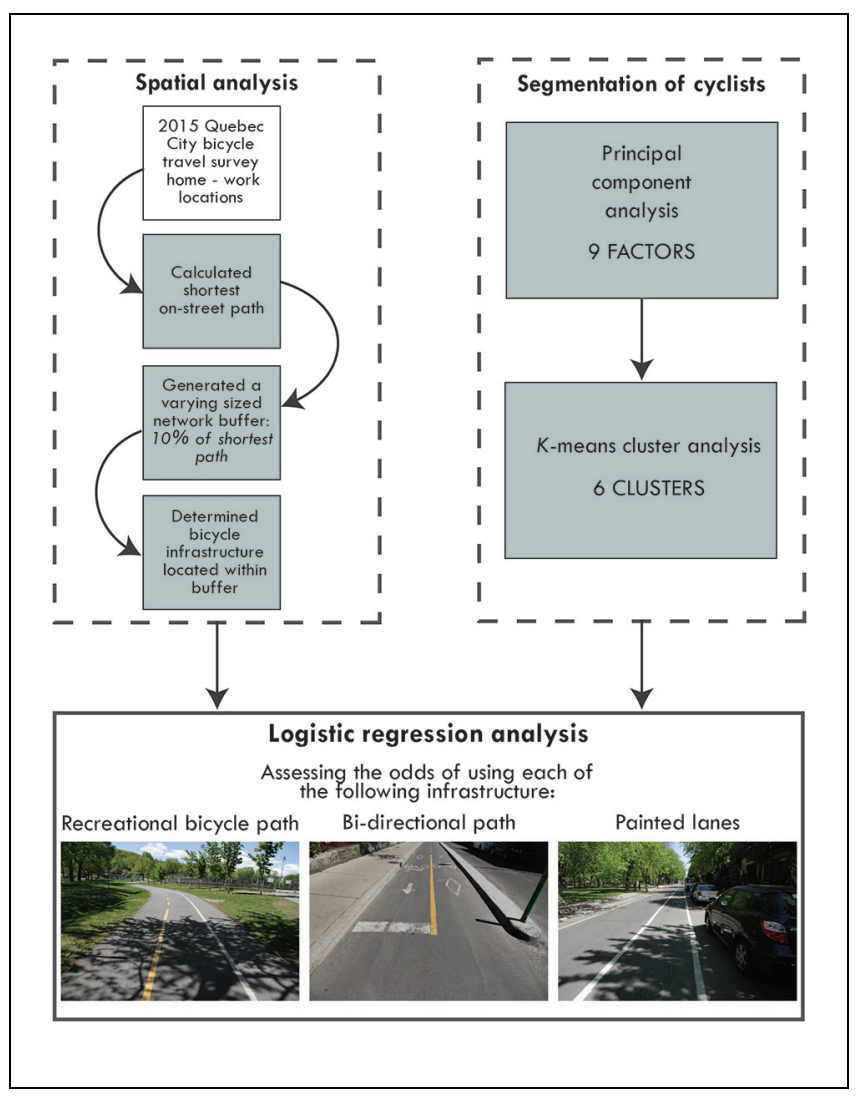

Figure 2. Analysis approach. presented in Figure 3. A varying sized network buffer was chosen for this method, assuming that the longer the respondent's route, the more opportunities an individual will have to divert away from their shortest path.

To identify the types of bicycle facilities that a cyclist has reasonable access to when commuting to work or school, we spatially joined the aforementioned buffer shapefile with the 2015 Bicycle Network shapefile. The results of this operation were also used to determine the length of each cycling facility present within each buffer. A cycling facility was considered present within a buffer if the segment length was greater than $25 \mathrm{~m}$. We determined the 25-m threshold according to our study area bicycle network characteristics and by analyzing the spatial joint results. We thus ensured that a facility segment was long enough to be considered as a real potential option for cyclists to divert from their shortest route to use it. We thereafter compared which facility type each respondent has reasonable access to and compared this with whether or not each respondent reported using those previously identified facilities.

Finally, we calculated the ratio of the supply of bicycle facilities within each route buffer relative to the total kilometers of streets present within each respondent's network buffer. To do so we summed the length of all bicycle facilities located within one buffer and divided this by the sum of the street length present within the

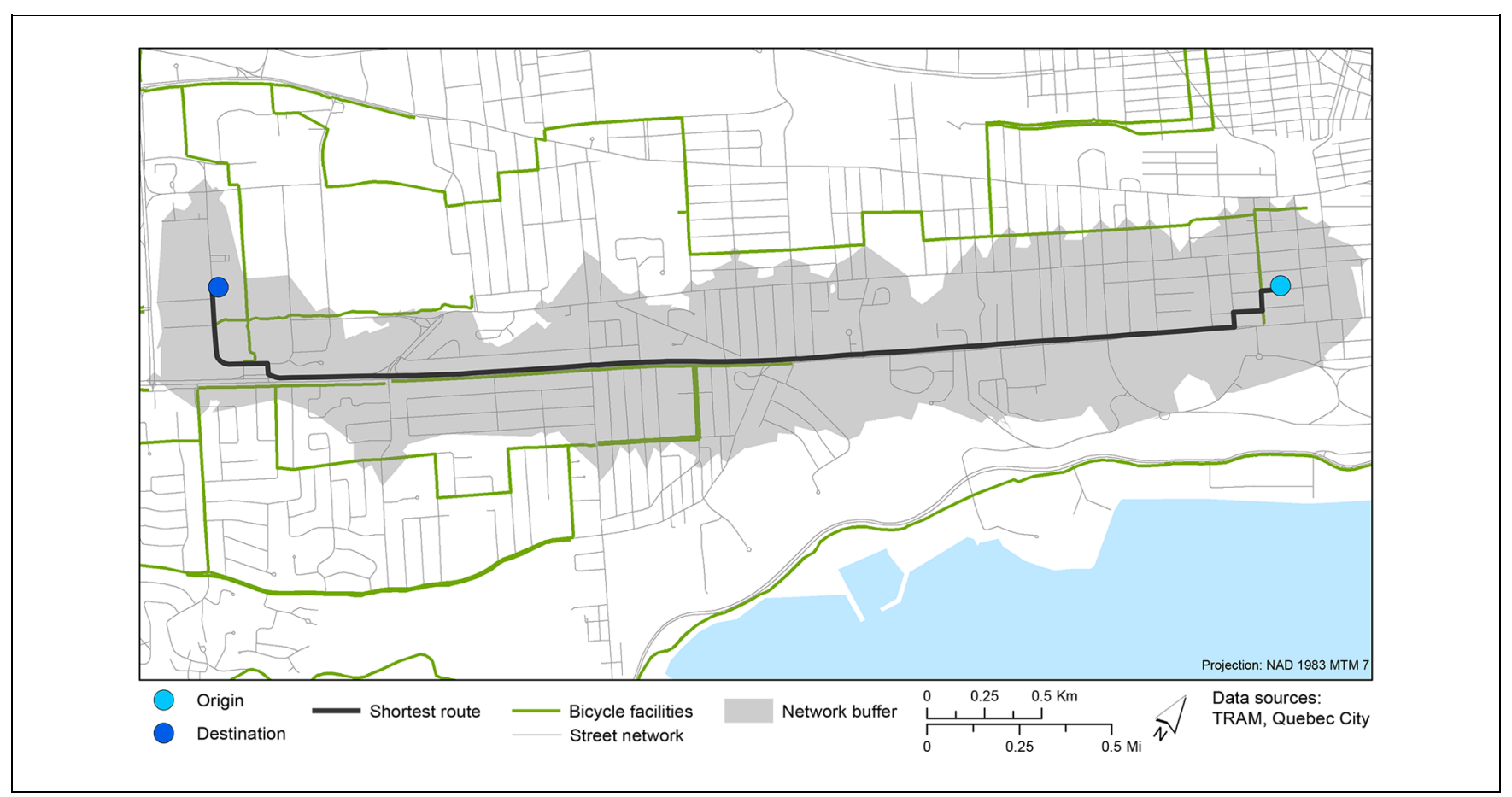

Figure 3. Example of a network buffer. 
Table 2. Results from the Principal Component Analysis

\begin{tabular}{|c|c|c|c|}
\hline \multicolumn{2}{|c|}{ Factors } & Variables & Loading \\
\hline I & Time efficiency & How important are these factors in your decision to cycle now? & \\
\hline & & I.I Flexibility for multiple trips & .850 \\
\hline & & I.2 Flexibility of my departure time & .849 \\
\hline & & I.3 It's the fastest way to get from $A$ to $B$ & .795 \\
\hline & & I.4 Predictability of travel time & .774 \\
\hline \multirow[t]{5}{*}{2} & Weather & I don't cycle when: & \\
\hline & & 2.I There is ice or snow because of the danger of slipping & .815 \\
\hline & & 2.2 There is snow because of the additional effort & .807 \\
\hline & & 2.3 lt's too cold & .573 \\
\hline & & 2.4 It's raining & .429 \\
\hline \multirow[t]{4}{*}{3} & Cycling is enjoyable & How important are these factors in your decision to cycle now? & \\
\hline & & 3.I Cycling is fun & .775 \\
\hline & & 3.2 It's part of my self-identity/culture & .762 \\
\hline & & 3.3 To what extent does cycling improve your quality life? & .618 \\
\hline \multirow[t]{3}{*}{4} & Effort & 4.I I don't cycle when the route I have to take is too steep & .752 \\
\hline & & 4.2 How important is a flat route in making a good bicycle route? & .705 \\
\hline & & 4.3 I don't cycle when I have to carry bags or heavy loads & .548 \\
\hline \multirow[t]{5}{*}{5} & Experience & 5.I As a child did you use a bicycle for getting around? & .710 \\
\hline & & 5.2 As a child did you use a bicycle for going to school? & .608 \\
\hline & & 5.3 Bicycles were seen as a common mode of transportation where I grew up & .514 \\
\hline & & 5.4 For how long have you been cycling regularly? & .488 \\
\hline & & 5.5 Did you start cycling as a child? & .449 \\
\hline \multirow[t]{2}{*}{6} & Family encouragement & $\begin{array}{l}\text { 6.I To what extent did your parent(s) or guardian(s) actively encourage or } \\
\text { discourage you to cycle as a sport or recreational activity? }\end{array}$ & .904 \\
\hline & & $\begin{array}{l}\text { 6.2 To what extent did your parent(s) or guardian(s) actively encourage or } \\
\text { discourage you to cycle as a way to reach destinations? }\end{array}$ & .881 \\
\hline \multirow[t]{2}{*}{7} & $\begin{array}{l}\text { Peer and institution } \\
\text { encouragement }\end{array}$ & $\begin{array}{l}\text { 7.I How important is your classmates/coworkers cycling in your decision to cycle } \\
\text { now? }\end{array}$ & .859 \\
\hline & & $\begin{array}{l}\text { 7.2 How important is encouragement from your employer/school in your } \\
\text { decision to cycle now? }\end{array}$ & .851 \\
\hline \multirow[t]{3}{*}{8} & Raised in the city & $\begin{array}{l}\text { 8.I Transit was seen as a common mode of transportation for most people } \\
\text { where I grew up }\end{array}$ & .696 \\
\hline & & 8.2 I grew up in an urban environment & .686 \\
\hline & & 8.3 Driving a car was a normal and important part of becoming an adult & .607 \\
\hline \multirow[t]{4}{*}{9} & Positive benefits & How important are these factors in your decision to cycle now? & \\
\hline & associated with & 9.1 Health & .704 \\
\hline & cycling & 9.2 Environment & .696 \\
\hline & & 9.3 Low cost of cycling & .524 \\
\hline
\end{tabular}

same buffer. A higher ratio indicates a greater presence of bicycle facilities in that buffer, meaning that cyclists have many bicycle facilities available to use when commuting to work or school by bicycle.

\section{Segmentation of Cyclists}

To segment our sample into distinct cyclist groups, we first conducted a factor analysis, namely a Principal Component Analysis (PCA), using the 2015 Bicycle Travel Survey. The data employed was derived from questions on satisfaction, motivation, and travel behavior. Note that we did not include questions related to infrastructure preferences as previous segmentation studies have, see (10) for example. For this reason, our results will differ from previous studies, while differences are also expected to arise between different cities. A PCA statistically examines the variance and covariance among a chosen set of survey question responses, revealing the structure of a dataset, and allowing the formation of factors, which are a group of responses that correlate among each other $(17,33)$. The PCA was operationalized in SPSS using varimax rotation and eigenvalues greater than 1 , to obtain, in a systematic fashion, an optimal number of factors.

A total of 29 variables were grouped together to create nine factors, which explained $59 \%$ of the variance of our selected data. Table 2 shows the results, where each variable is displayed with its respective loading. Note that a loading closer to 1 indicates a stronger relationship between a variable and its factor.

In a second step, we conducted a $K$-means cluster analysis using the factors previously generated. This technique classified our sample into clusters or distinct 


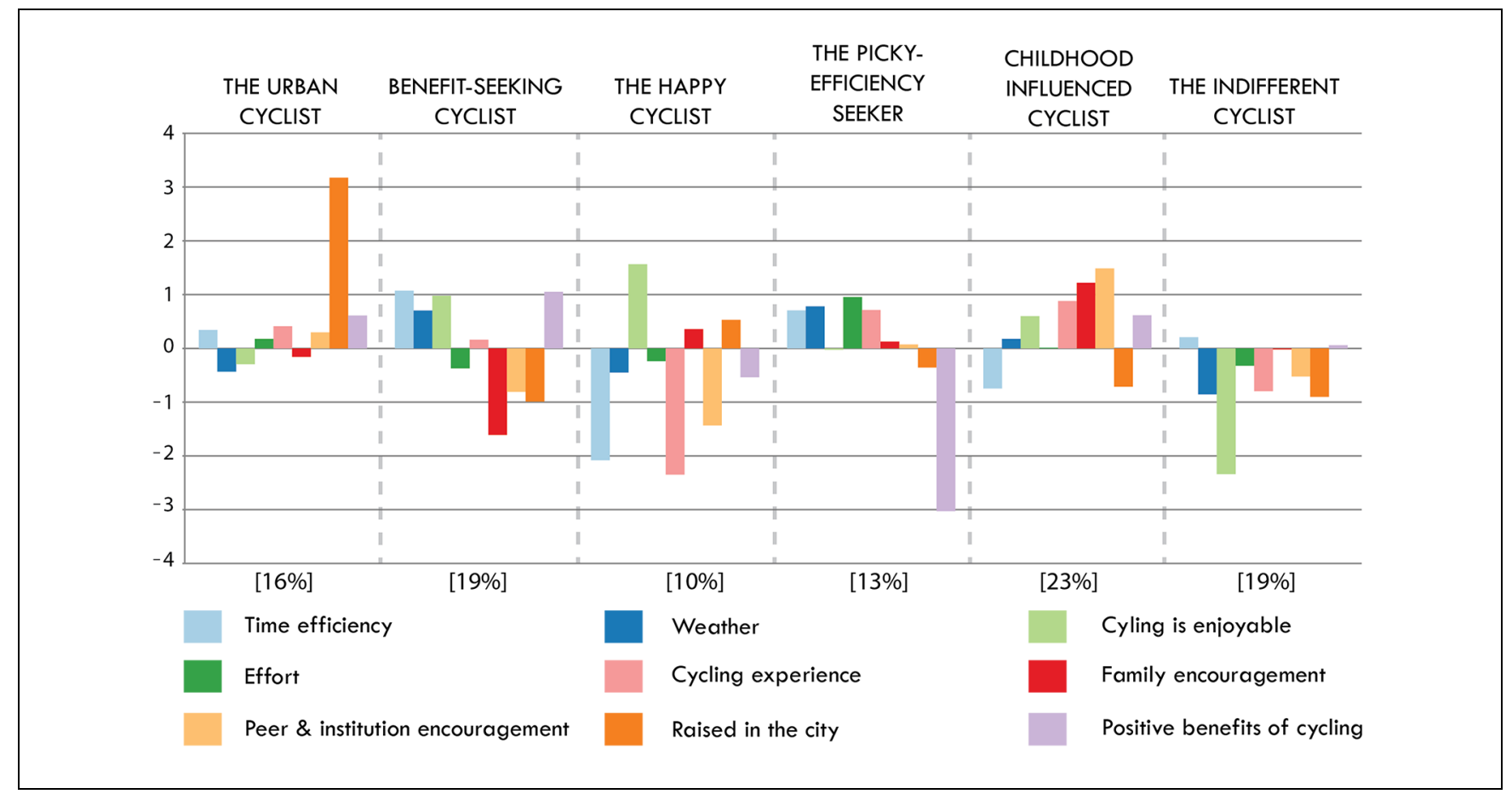

Figure 4. Cyclist segmentation derived from factor and cluster analysis.

groups of survey respondents, where the differences between each group are maximized, while at the same time favoring similarities within members of the same group $(10,17)$. The final number of cyclist types was determined in an iterative fashion by evaluating the outcomes of different grouping options ranging from three to seven clusters. Determining the final number of clusters was guided by the following four factors as previously recommended by Krizek and El-Geneidy (34): (1) statistical output; (2) transferability to transport policy; (3) lessons from previous research; and (4) common sense and intuition.

Figure 4 presents our cyclist segmentation composed of the six following clusters: (1) the urban cyclist; (2) the benefit-seeking cyclist; (3) the happy cyclist; (4) the picky efficiency seeker; (5) the childhood-influenced cyclist; and (6) the indifferent cyclist. The colored bars represent the loading of each factor and indicate to what extent each cyclist perceived that factor either positively or negatively relative to other clusters. The types of cyclists were named according to their most salient characteristics, which are described in the following section.

The urban cyclist- $16 \%$ of the sample - is characterized by the predominance of individuals $(75 \%)$ growing up in an urban environment. On average, they cycle 6.8 $\mathrm{km}$ to reach their workplace or school location. The majority of Urban Cyclists (71\%) perceived transit as a common mode of transport when growing up and 33\% believed that driving a car was a normal and important part of becoming an adult. Urban Cyclists are also slightly more motivated by the positive benefits associated with cycling to work than most of the other groups of cyclists. Furthermore, poor weather conditions, such as ice, are less likely to negatively affect their decision to cycle. They are fairly neutral regarding the importance of peer and institutional encouragement as well as physical efforts required while cycling.

The benefit-seeking cyclist- $19 \%$ of the sample-is motivated above all by the benefits associated with cycling to work or school. The environmental and health benefits, as well as the low cost of cycling appear important to them. Their decision to cycle is also influenced by their perception of cycling as being time efficient. Similar to urban cyclists, they cycle on average $6.5 \mathrm{~km}$ to reach their workplace or school location. The benefit-seeking cyclist perceives cycling as enjoyable and seems rather unbothered by encumbrances and route steepness. However, they prefer not to cycle in poor weather conditions, especially when there is ice or snow. Finally, in their childhood, these cyclists were fairly discouraged by their parents or guardians from using a bicycle to reach a destination. Interestingly, $61 \%$ of this group grew up in a suburban environment.

The happy cyclist- $10 \%$ of the sample - perceived cycling as an enjoyable mode of transport and as part of their self-identity. Their decision to cycle is positively influenced by the idea that cycling can improve their quality of life. Interestingly, happy cyclists cycle on 
average $8.2 \mathrm{~km}$ to reach their destination, which corresponds to the greatest average commute distance of all groups. Nearly $84 \%$ of this group began to cycle as a child. However, in their childhood, only $30 \%$ used their bicycle to get around and $13 \%$ cycled to school. In fact, this group received moderate encouragement from their family or guardians to cycle for utilitarian and recreational purposes. Finally, they do not particularly value peer and institutional encouragement and they give the least importance to travel time predictability in their decision to cycle.

The picky efficiency seekers- $13 \%$ of the samplecycle to work mainly for efficiency and practical reasons, and under certain conditions. In fact, time savings positively influence their decision to cycle; however, this group is least likely to cycle in poor weather conditions and when the effort required to reach their destination is perceived as too high. Picky efficiency seekers are also the least motivated by the benefits of cycling. In addition, they are somewhat neutral toward the joy of cycling and encouragement. Finally, they cycle on average 6.1 $\mathrm{km}$ to reach their workplace or school location and have been cycling regularly for the longest period of time among all groups. Nearly, $20 \%$ of this group grew up in an urban environment.

The childhood-influenced cyclists-23\% of the sample - all began to cycle as children and were highly encouraged by family or guardians to cycle for recreational and utilitarian purposes. In their childhood, nearly $80 \%$ of childhood-influenced cyclists cycled to get around and slightly more than half of this group used their bicycles to get to school. Interestingly, $44 \%$ of this group perceived cycling as a common mode of transport when growing up and around $70 \%$ were raised in the suburbs. Overall, childhood-influenced cyclists perceived cycling as enjoyable. On average, they cycle $4.9 \mathrm{~km}$ to reach their workplace or school. Finally, the benefits of cycling are important in these individuals' decision to cycle. They are also neutral about efforts required to reach their destination and poor weather conditions.

The indifferent cyclists- $19 \%$ of the sample - are neutral about cycling benefits and unbothered by factors that could negatively affect their decision to cycle. On average, this group cycles $3.6 \mathrm{~km}$ to reach their destination, which is the shortest average commuting distance of all groups. In fact, indifferent cyclists are not discouraged by the efforts required to reach their destination and by poor weather conditions, and yet, they do not associate themselves with the cycling culture. In a similar vein, this group is the least motivated by the idea that cycling is enjoyable. They are slightly motivated by time efficiency and are rather neutral toward the benefits of cycling. Finally, nearly $70 \%$ of this group grew up in a suburban environment, where cycling was not perceived as a common form of transport.

Next, we examined the six types of cyclists described above according to their reported facility type usage and access to each facility type on their daily commute (Table 3). More than half of the cyclists reported using a recreational path and a painted lane when commuting to work or school, while just a third of them reported using a bi-directional path. Interestingly, nearly $57 \%$ have reasonable access to a bi-directional path, but did not report using it. This finding indicates that the majority of cyclists whose commuting route is in proximity to a bi-directional path decided not to cycle on this type of facility. This also suggests that there are possibly designrelated factors pertaining to bi-directional paths in Quebec City that deter cyclists from using them. In comparison, only a third of all cyclists who have reasonable access to recreational paths and painted lanes did not report using them.

\section{Logistic Regression Analysis}

To predict the odds that each individual, previously classified in a cyclist type, uses a recreational path, bidirectional path, or painted lane, we constructed three binary logistic regression models, one for each facility type. For each model, the dependent variable was derived from the following question: "When you travel to work/ school by bicycle, do you usually use the type of facility shown above?" The dependent variable employed is a dummy that equals 1 if a respondent reported using a facility type and 0 if a respondent reported not using a facility type. Additionally, we controlled for trip, neighborhood, and personal characteristics.

Table 4 presents the results of the three binary logistic regressions. Holding all other variables constant, Model 1 shows that the likelihood of using a recreational path to commute to work or school is 3.49 times higher for a cyclist who has reasonable access to this facility type than for those who have not. Model 2 uncovers that the likelihood of using a bi-directional path is 1.42 times higher when cyclists have reasonable access to this facility type than when they do not. However, this finding is not statistically significant. So, having reasonable access to a bidirectional bicycle path is not a predictor of whether or not an individual will use that facility. While we assumed that all cyclists could potentially divert from their shortest route up to $10 \%$ of their total trip distance, further analysis could be conducted to test different diversion rate options. In Model 3, the odds of using a painted lane are 1.72 times higher for cyclists who have reasonable access to this facility type, holding all other variables at their mean. 


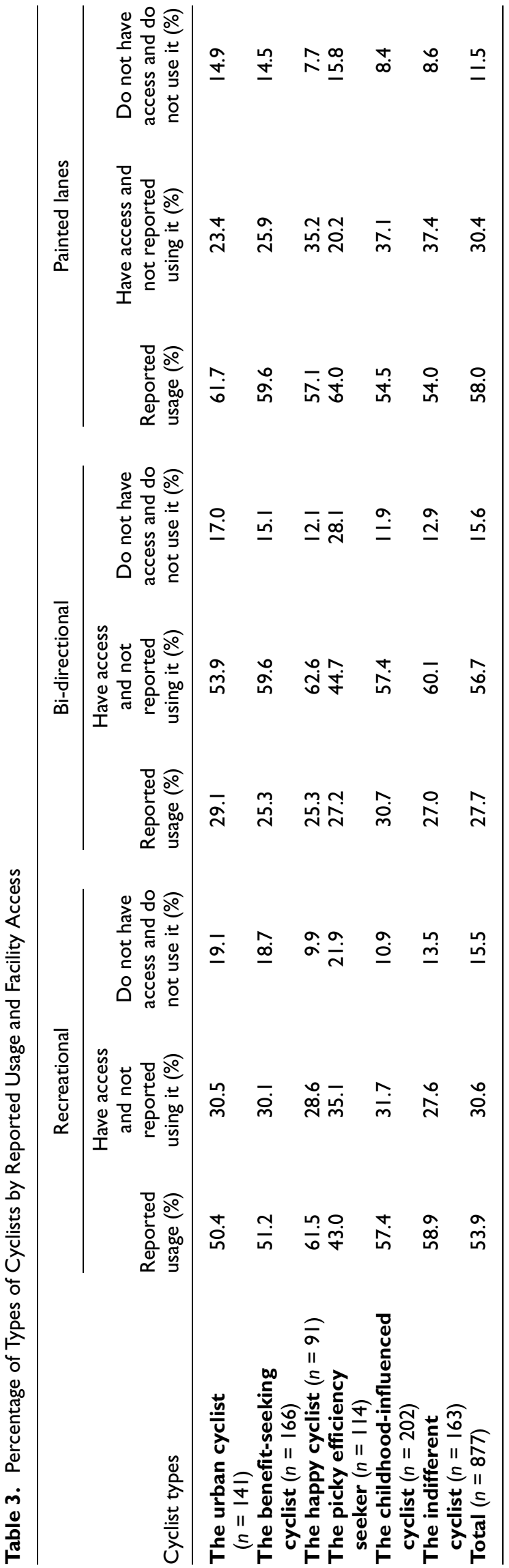

The results of these three models could be explained by the fact that cyclists commuting to work or school are more likely to be traveling during morning and evening peak hours, a period characterized by heavy motorized flow as mentioned by Broach, Dill and Gliebe (8). While over half of bi-directional paths and painted lanes are adjacent to roads with a speed limit of $60 \mathrm{~km} / \mathrm{h}$ or above, cyclists could be more willing to use recreational paths, when having access to them, as they are located further away from car traffic. In addition, cyclists using recreational paths to reach their destination cross fewer street intersections, which could eventually reduce their travel time. Future analysis could include cyclists' perceptions of bicycle facility safety and comfort.

Cycling Segmentation. Model 1 reveals that the odds of indifferent cyclists using recreational paths when commuting to work is 2.13 times higher compared with picky efficiency seekers, all else equal. Indifferent cyclists are defined as being rather neutral and unbothered by factors that could affect their decision to cycle, such as poor weather conditions or positive benefits of cycling, while picky efficiency seekers are mainly cycling for efficiency reasons.

Model 2 indicates that there is no statistically significant difference in the odds of using a bi-directional path between picky efficiency seekers and all other types of cyclists. This is rather surprising giving the existing literature on how different types of cyclist have specific preferences in bicycle facilities. Note that, across all cyclist types, between $45 \%$ and $63 \%$ of cyclists reported having access to a bi-directional path but are not using it to commute. Thereafter, the design and locations of bidirectional bicycle paths in Quebec City should be thoroughly examined to shed light on this finding.

In Model 3, the odds of childhood-influenced cyclists using painted lanes are $47 \%$ lower compared with picky efficiency seekers, when keeping all other variables at their mean. Similarly, happy cyclists and indifferent cyclists are $41 \%$ and $39 \%$ less likely to use painted lanes compared with picky efficiency seekers, although this finding is statistically significant at the $90 \%$ level.

Overall, this study uncovers subtle differences in bicycle facility usage between our sample of cyclist types. As previous cyclist typologies (for example, DamantSirois, Grimsrud, and El-Geneidy (10)) included infrastructure preferences in their segmentation process, we cannot compare our results with previous studies that observe distinct infrastructure preferences among certain cyclists.

Trip, Neighborhood, and Personal Characteristics. Commuting trip distance positively influences the odds of cycling on all facility types. For every additional kilometer cycled, 
Table 4. Likelihood of Using Each Bicycle Facility

\begin{tabular}{|c|c|c|c|c|c|c|c|c|c|}
\hline \multirow{3}{*}{$\begin{array}{l}\text { Variable } \\
\begin{array}{l}\text { Presence of infrastructure } \\
\text { within route buffer }\end{array}\end{array}$} & \multicolumn{3}{|c|}{$\begin{array}{c}\text { Model I } \\
\text { Recreational path }\end{array}$} & \multicolumn{3}{|c|}{$\begin{array}{c}\text { Model } 2 \\
\text { Bi-directional path with median }\end{array}$} & \multicolumn{3}{|c|}{$\begin{array}{c}\text { Model } 3 \\
\text { Painted lane }\end{array}$} \\
\hline & \multirow{2}{*}{$\frac{\text { Odds ratio }}{3.49 * * *}$} & \multicolumn{2}{|c|}{ Conf. interval } & \multirow{2}{*}{$\begin{array}{c}\text { Odds ratio } \\
1.42\end{array}$} & \multicolumn{2}{|c|}{ Conf. interval } & \multirow{2}{*}{$\frac{\text { Odds ratio }}{1.72 * *}$} & \multicolumn{2}{|c|}{ Conf. interval } \\
\hline & & 2.30 & 5.37 & & -0.17 & 0.91 & & 0.09 & 0.99 \\
\hline \multicolumn{10}{|l|}{ Cyclist segmentation } \\
\hline I. The urban cyclist ${ }^{\mathrm{a}}$ & 1.26 & 0.73 & 2.17 & 1.05 & -0.53 & 0.64 & 0.84 & -0.72 & 0.36 \\
\hline 2. The benefit-seeking cyclist ${ }^{a}$ & 1.40 & 0.83 & 2.37 & 0.91 & -0.67 & 0.48 & 0.73 & -0.83 & 0.20 \\
\hline 3. The happy cyclist $^{\mathrm{a}}$ & 1.56 & 0.85 & 2.90 & 0.69 & -1.05 & 0.29 & $0.59 *$ & -1.13 & 0.06 \\
\hline $\begin{array}{l}\text { 5. The childhood-influenced } \\
\text { cyclist }^{\mathrm{a}}\end{array}$ & $\mathrm{I} .45$ & 0.87 & 2.42 & 0.98 & -0.56 & 0.53 & $0.53 * *$ & -1.13 & -0.13 \\
\hline $\begin{array}{l}\text { 6. The indifferent cyclist }{ }^{\mathrm{a}} \\
\text { Ref: The picky efficiency seeker }{ }^{\mathrm{a}} \\
\text { Trip and neighborhood } \\
\text { characteristics }\end{array}$ & $2.13 * * *$ & 1.26 & 3.63 & 1.10 & -0.48 & 0.67 & $0.61 *$ & -1.03 & 0.01 \\
\hline $\begin{array}{l}\text { Length of work/school commute } \\
(\mathrm{km})\end{array}$ & $1.08 * * *$ & 1.04 & 1.12 & $1.10 * * *$ & 0.06 & 0.13 & $1.05 * *$ & 0.01 & 0.08 \\
\hline $\begin{array}{l}\text { Ratio of bicycle facilities to } \\
\text { street length within route } \\
\text { buffers }\end{array}$ & $1.04 * *$ & 1.00 & 1.07 & $1.04 * *$ & 0.00 & 0.08 & $1.06 * * *$ & 0.02 & 0.09 \\
\hline $\begin{array}{l}\text { Perceived neighborhood as } \\
\text { cycle-friendly in relation to } \\
\text { infrastructure }^{\mathrm{a}}\end{array}$ & $2.05 * * *$ & 1.52 & 2.77 & $1.47 * *$ & 0.07 & 0.70 & 0.92 & -0.37 & 0.20 \\
\hline \multicolumn{10}{|l|}{ Personal characteristics } \\
\hline Age below 35 years old ${ }^{a}$ & $0.63 *$ & 0.39 & 1.01 & 0.72 & -0.80 & 0.16 & 0.90 & -0.56 & 0.34 \\
\hline $\begin{array}{l}\text { Age between } 35 \text { and } 54 \text { years } \\
\text { old }^{\mathrm{a}}\end{array}$ & 0.74 & 0.47 & 1.18 & 0.68 & -0.85 & 0.09 & 1.05 & -0.40 & 0.49 \\
\hline \multicolumn{10}{|l|}{ Ref Age 64 years and above } \\
\hline Gender-Female ${ }^{\mathrm{a}}$ & 0.92 & 0.68 & 1.24 & $0.57 * * *$ & -0.90 & -0.22 & 0.89 & -0.40 & 0.18 \\
\hline
\end{tabular}

Note: Conf. $=$ confidence interval. Dependent variable: Reported usage $(\mathrm{I}=$ used and $0=$ not used $)$.

${ }^{\text {a }}$ Represents a binary dummy variable.

*90\% significance level; **95\% significance level; ***99\% significance level.

the odds of using recreational, bi-directional, and painted lanes increase by $8 \%, 10 \%$, and $5 \%$ respectively. This finding suggests that cyclists commuting longer distances to work are more likely to use an available bicycle facility on at least part of their route. With respect to the supply of bicycle facilities within a cyclist's buffer relative to total length of street, we observe that a larger ratio of bicycle facilities to street length is associated with a $4 \%$ increase in odds of using a recreational path and bidirectional path with a median, and a $6 \%$ increase in the odds of using a painted lane. Our results also reveal that respondents who perceived their neighborhood as cyclefriendly in relation to bicycle infrastructure were more likely to have reported using a recreational path and a bi-directional path. However, no significant difference was observed for the use of painted lanes. This suggests that if cycling infrastructure investments in a respondents' neighborhood are perceived as unsatisfactory, cyclists are less likely to use that infrastructure, even if it is within a reasonable distance of their commuting route. Alternatively, this variable may capture the influence of self-selection, whereby individuals have chosen to live in a neighborhood with access to these types of bicycle facilities and are therefore more likely to use them on their daily commute.

Finally, across all facility types, age is not a statistically significant predictor of facility use, with one exception. Cyclists under the age of 35 were found to be $37 \%$ less likely to use a recreational path compared with cyclists over the age of 55. The lack of significance across age was unsurprising given previous literature that has found inconsistent findings for preferences for separated infrastructure across age groups (27). With respect to gender differences, women are $43 \%$ less likely to use a bidirectional path compared with men. However, no differences were observed for the other two facility types. This result may be related to the design of the bi-directional paths in Quebec City and may indicate that the design of this facility type may invoke feelings of stress or safety concerns that are more pronounced among female cyclists. Female cyclists have previously been found to feel more unsafe than males under similar traffic 
conditions and have attached greater importance to safety concerns (including traffic speed and volume, signalized intersections etc.) relative to men (35), which may explain the lower usage of this facility type among women.

\section{Conclusions and Recommendations}

The objective of this study was to present a methodology to assess how distinct types of cyclists are using, or not using, three types of bicycle facilities: recreational paths, bi-directional paths, and painted bicycle lanes. Using the 2015 Quebec City Bicycle Survey, the first part of this analysis consisted of routing each respondent's commute trip and determining what bicycle facilities each respondent had access to along their route, assuming that cyclists are willing to divert from the shortest path to use a preferred cycling facility. We created personalized network buffers around the shortest route connecting each cyclist's home and work/school location, where the buffer size was created according to the distance of each respondent's commute. Accordingly, as a cyclist is traveling a greater distance, the area that is deemed reasonable to divert within is also wider and can potentially encompass more cycling facilities. Using these buffers, we identified which cycling facility types each respondent had access to in order to discern who is or is not using bicycle facilities that are available to them.

To our knowledge, our study is the first to employ this personalized buffer method to account for cyclists' willingness to divert from their shortest route to reach a preferred cycling facility. We recommend to practitioners wishing to reproduce and adapt this method to try different diversion rates according to their city context; shorter diversion rates have been observed in cities or regions with a large supply of bicycle facilities (21). Future studies could also examine the impact of using different diversion rates on their results.

In the second part of the analysis, we segmented our sample into six distinct types of cyclists: the urban cyclist; the benefit-seeking cyclist; the happy cyclist; the picky efficiency seeker; the childhood-influenced cyclist; and the indifferent cyclist. We derived our cyclist typology from factors such as their motivations, childhood characteristics, and sensitivity to peer and family encouragement. In the final phase of our analysis, we constructed three logistic regression models to determine the odds that a cyclist will use each facility type, controlling for whether or not that facility type was available to them on their commute to work or school. The models indicate that in Quebec City, if a cyclist has access to all three facility types on their commute, that same cyclist is most likely to use a recreational path, followed by a painted bicycle lane. Interestingly, we found that access to a bi- directional path on a respondent's commute trip was not a predictor of their reported usage of this facility type. In fact, the majority $(57 \%)$ of cyclists in our sample have access to a bi-directional path on their commute trip and did not report using it while responding to the survey. As the data used in this study are a subset of all cyclists in Quebec City, it is difficult to prove or disprove if our study sample generally represents the cycling population of Quebec City in relation to their decision making about trips in the absence of comprehensive knowledge of the cyclist route choice in the region from other sources. Nonetheless, the results of this analysis are consistent with the literature and local experience. Furthermore, we must keep in mind that because a facility type in Quebec City is not well used, it does not necessarily mean that this facility is poorly perceived in our study area or elsewhere, and that it will not be desired in other urban contexts. In reality, the location of a bicycle facility may not effectively connect cyclists with their destinations; diverting from their shortest route may not, therefore, constitute an optimal option for some cyclists.

Modest differences were observed among the odds of using a bicycle facility type across our cyclist typology. In comparison, Misra and Watkins (31) observed that cyclist typology, derived from a cyclist's comfort level, did not predict a cyclist's decision to deviate from the shortest route to use a bicycle facility; however, the authors did not consider the type of bicycle facility in their analysis. In our study, with reference to the picky efficiency seekers (highly motivated by time efficiency) all other types of cyclists were more likely to use recreational paths on their commute, while less likely to use painted lanes. This finding suggests that these cyclists are unlikely to deviate far from their shortest path, as a detour is typically required to use a recreational path; however, knowledge of these cyclists' actual routes would allow us to confirm this. Knowledge of the types of cyclist in a region, including their motivations (i.e., time efficiency) and deterrents (i.e., cycling on high vehicle volume streets) should guide planners toward the optimal facility design in that region.

Irrespective of type, cyclists in our study sample have a strong preference for the use of off-street recreational paths, which is the facility type that offers the greatest separation from traffic in Quebec City. This is consistent with several studies that have observed cyclists' preferences for facilities with greater separation from traffic (2, 4, 26). However, the other physically separated bicycle facility type in our study, the bi-directional path, which is a common facility design in Quebec City and other cities including Montreal, appears to contradict previous literature. While these facilities are physically separated from traffic, and therefore may offer greater protection to cyclists than, for example painted lanes, the bi-directional 
design of these lanes may be detrimental to how cyclists perceive their safety. In Montreal, Damant-Sirois, Grimsrud, and El-Geneidy (10) observed that painted lanes running in the opposite direction to traffic are the least preferred cycling facilities in the city. Accordingly, future studies should verify how cycling usage differs between physically separated bi-directional and unidirectional lanes in a city where both types are available. In another respect, $52 \%$ of bi-directional lanes in Quebec follow streets with motorized speeds limits of $60 \mathrm{~km} / \mathrm{h}$, which may explain the lower than expected usage of these facilities. In the future, we recommend monitoring intersections along bi-directional lanes to assess interactions between cyclists, vehicles, and pedestrians.

Our results highlight the importance of thinking critically about what type of bicycle infrastructure is preferable according to a specific urban context and the typology of cyclists present in a region. Expanding or incrementally improving an existing cycling network should be achieved by considering the network holistically, and not solely by deciding on the facility type or design to implement (4). As such, not only facility design but also characteristics of adjacent streets, and neighborhood characteristics should be considered when deciding which facility type is best suited (4). Moreover, given the diversity in cycling facility preferences, planners should engage in a dialog with cyclists, both novice and more experienced cyclists, to collect information about safety and stress levels when using different facilities, with the goal of identifying optimal cycling facilities for future investments.

\section{Acknowledgments}

The authors wish to thank Mr. Jean-François Martel, transportation planner at the City of Quebec, who provided the municipal bicycle shapefile data. This study was funded by the Social Sciences and Humanities Research Council of Canada and the Natural Sciences and Engineering Research Council of Canada.

\section{Author Contributions}

The authors confirm contribution to the paper as follows: study conception and design: M-PV, EG, AE-G; data collection: MPV, EG, AE-G; analysis and interpretation of results: M-PV, EG, AE-G; draft manuscript preparation M-PV, EG, AE-G. All authors reviewed the results and approved the final version of the manuscript.

\section{References}

1. Pucher, J., J. Dill, and S. Handy. Infrastructure, Programs, and Policies to Increase Bicycling: An International Review. Preventive Medicine, Vol. 50, 2010, pp. S106-S125.

2. Winters, M., G. Davidson, D. Kao, and K. Teschke. Motivators and Deterrents of Bicycling: Comparing Influences on Decisions to Ride. Transportation, Vol. 38, 2011, pp. $153-168$.

3. Handy, S., and Y. Xing. Factors Correlated with Bicycle Commuting: A Study of Six Small U.S. Cities. International Journal of Sustainable Transportation, Vol. 5, No. 2, 2011, pp. 91-110.

4. Buehler, R., and J. Dill. Bikeway Networks: A Review of Effects on Cycling. Transport Reviews, Vol. 36, No. 1, 2016, pp. 9-27.

5. Wardman, M., M. Tight, and M. Page. Factors Influencing the Propensity to Cycle to Work. Transportation Research Part A, Vol. 41, 2007, pp. 339-350.

6. Nkurunziza, A., M. Zuidgeest, and M. Van Maarseven. Examining the Potential Modal Change: Motivators and Barriers for Bicycle Commuting in Dar-es-Salaam. Transport Policy, Vol. 24, 2012, pp. 249-259.

7. Heinen, E., K. Maat, and B. van Wee. The Role of Attitude Toward Characteristics of Bicycle Commuting on the Choice to Cycle to Work Over Various Distances. Transportation Research Part D, Vol. 16, 2011, pp. 102-109.

8. Broach, J., J. Dill, and J. Gliebe. Where Do Cyclists Ride? A Route Choice Model Developed with Revealed Preference GPS Data. Transportation Research Part A, Vol. 46, 2012, pp. 1730-1740.

9. Tilahun, N. Y., D.M. Levinson, and K. J. Krizek. Trails, Lanes, or Traffic: Valuing Bicycle Facilities with an Adaptative Stated Survey. Transportation Research Part A, Vol. 41, 2007, pp. 287-301.

10. Damant-Sirois, G., M. Grimsrud, and A. El-Geneidy. What's Your Type: A Multidimensional Cyclist Typology. Transportation, Vol. 41, No. 6, 2014, pp. 1153-1169.

11. Dill, J., and N. McNeil. Four Types of Cyclists ? Examination of Typology for Better Understanding of Bicycling Behavior and Potential. Transportation Research Record: Journal of the Transportation Research Board, 2013. 2387: 129-138.

12. Dill, J., and N. McNeil. Revisiting the Four Types of Cyclists. Findings from a National Survey. Transportation Research Record: Journal of the Transportation Research Board, 2016. 2587: 90-99.

13. Geller, R. Four Types of Cyclists. Portland Office of Transportation, Ore., 2006.

14. Eriksson, L., M. Friman, and T. Gärling. Perceived Attributes of Bus and Car Mediating Satisfaction with the Work Commute. Transportation Research Part A, Vol. 47, 2013, pp. 87-96.

15. Handy, S., B. van Wee, and M. Kroesen. Promoting Cycling for Transport: Research Needs and Challenges. Transport Reviews, Vol. 34, No. 1, 2014, pp. 4-24.

16. Anable, J. 'Complacent Car Addicts' or 'Aspiring Environmentalists'? Identifying Travel Behaviour Segments Using Attitude Theory. Transport Policy, Vol. 12, No. 1, 2005, pp. 65-78.

17. Grisé, E., and A. El-Geneidy. Where Is the Happy Transit Rider? Evaluating Satisfaction with Regional Rail Service Using a Spatial Segmentation Approach. Transportation Research Part A: Policy and Practice, Vol. 114, 2017, pp. 84-96. 
18. Gatersleben, B., and H. Haddad. Who Is the Typical Bicyclist? Transportation Research Part F: Traffic Psychology and bBehaviour, Vol. 13, No. 1, 2010, pp. 41-48.

19. Broach, J., J. Gliebe, and J. Dill. Development of a MultiClass Bicyclist Route Choice Model Using Revealed Preference Data. Presented at International Conference on Travel Behavior Research, Jaipur, India, 2009.

20. Krizek, K., A. El-Geneidy, and K. Thompson. A Detailed Analysis of How an Urban Trail System Affects Cyclists' Travel. Transportation, Vol. 34, 2007, pp. 611-624.

21. Larsen, J., and A. El-Geneidy. A Travel Behavior Analysis of Urban Cycling Facilities in Montréal, Canada. Transportation Research Part D: Transport and Environment, Vol. 16, No. 2, 2011, pp. 172-177.

22. Vedel, S. E., J. B. Jacobsen, and S.-P. Hans. Bicyclist' Preferences for Route Characteristics and Crowding in Copenhagen- A Choice Experiment Study of Commuters. Transportation Research Part A, Vol. 100, 2017, pp. 53-64.

23. Winters, M., K. Teschke, M. Grant, E. Setton, and M. Brauer. How Far Out of the Way Will We Travel? Built Environnement Influences on Route Selection for Bicycle an Car Travel. Transportation Research Record: Journal of the Transportation Research Board, 2010. 2190: 1-10.

24. Akar, G., and J. Clifton, K. . Influence of Individual Perceptions and Bicycle Infrastructure on Decision to Bike. Transportation Research Record: Journal of the Transportation Research Board, 2009. 2140: 165-172.

25. Sener, I., N. Eluru, and C. Bhat. An Analysis of Bicycle Route Choice Preferences in Texas, US. Transportation, Vol. 36, No. 5, 2009, 511-539.

26. Broach, J., and J. Dill. Using Predicted Bicyclist and Pedestrian Route Choice to Enhance Mode Choice Models. Transportation Research Record: Journal of the Transportation Research Board, 2016. 2564: 52-59.

27. Aldred, R., B. Elliott, J. Woodcock, and A. Goodman. Cycling Provision Separated from Motor Traffic: A
Systematic Review Exploring Whether Stated Preferences Vary by Gender and Age. Transport Reviews, Vol. 37, No. 1, 2017, pp. 29-55.

28. Goodman, A., J. Green, and J. Woodcock. The Role of Bicycle Sharing Systems in Normalising the Image of Cycling: An Observational Study of London Cyclists. Journal of Transport \& Health, Vol. 1, No. 1, 2014, pp. 5-8.

29. Howard, C., and E. Burns. Cycling to Work in Phoenix: Route Choice, Travel Behavior, and Commuter Characteristics. Transportation Research Record: Journal of the Transportation Research Board, 2001. 1773: 39-46.

30. Hunt, J., and J. Abraham. Influences on Bicycle Use. Transportation, Vol. 34, No. 4, 2007, pp. 453-470.

31. Misra, A., and K. Watkins. Modeling cyclists' Willingness to Deviate from Shortest Path Using Revealed Preference Data. Presented at 96th Annual Meeting of the Transportation Research Board, Washington, D.C., 2017.

32. Boisjoly, G., and A. El-Geneidy. Are We Connected? Assessing Bicycle Network Performance Through Directness and Connectivity Measures, a Montreal, Canada Case Study. Presented at 95th Annual Meeting of the Transportation Research Board, Washington, D.C., 2016.

33. Washington, S., M. Karlaftis, and F. Mannering. Statistical and Econometric Methods for Transportation Data Analysis. CRC Press, Boca Raton, Fla., 2011.

34. Krizek, K., and A. El-Geneidy. Segmenting Preferences and Habits of Transit Users and Non-Users. Journal of Public Transportation, Vol. 10, No. 3, 2007, pp. 71-94.

35. Berggren, M., A. Graves, H. Pikus, and L. Wirtis. The $20 \mathrm{~s}$ Bikeway: Clinton to Steele. Portland State University, 2012.

The Standing Committee on Bicycle Transportation (ANF20) peer-reviewed this paper (19-00378). 